\title{
Recent Advances in Diagnosis and Treatment for Malignancies of the Gastrointestinal Tract
}

\author{
Masayuki Watanabe ${ }^{a}$ Hideo Baba $^{a}$ Chikashi Ishioka ${ }^{b}$ Yasumasa Nishimura ${ }^{c}$ \\ Manabu Muto ${ }^{d}$ \\ ${ }^{a}$ Department of Gastroenterological Surgery, Graduate School of Medical Sciences, Kumamoto University, \\ Kumamoto, ${ }^{b}$ Department of Clinical Oncology, Research Institute of Development, Aging and Cancer, \\ Tohoku University, Sendai, ' Department of Radiation Oncology, Kinki University School of Medicine, Sayama, and \\ ${ }^{\mathrm{d} D e p a r t m e n t}$ of Gastroenterology and Hepatology, Graduate School of Medicine, Kyoto University, Kyoto, Japan
}

\section{Key Words}

Gastrointestinal cancer • Less invasive treatment •

Multimodal treatment

\begin{abstract}
Recent advances in diagnostic imaging have made it possible to detect early cancers in the gastrointestinal tract, while the development of novel antitumor agents has contributed to improved survival of patients with advanced cancers. In this review, the contents of the core symposia on 'Recent Advances in Diagnosis and Treatment for Malignancies of the Gastrointestinal Tract', held at the 6th and 7th annual meeting of The Japanese Gastroenterological Association, are summarized. At the 6 th annual meeting the core symposium focused on 'Progress in Chemotherapy and Targeted Therapy for Gastrointestinal Malignancies'. On the other hand, the 7th annual meeting focused on 'Recent Advances in Diagnosis and Treatment for Esophageal Cancer'. Less invasiveness and multidisciplinary approach are the two major key words in the management of gastrointestinal malignancies. Efforts in translational research as well as in clinical studies will deliver less invasive and more effective treatment for gastrointestinal cancers.

Copyright $\odot 2012$ S. Karger AG, Basel
\end{abstract}

\section{Introduction}

The Japanese Gastroenterological Association (JGA) organizes core symposia at the annual scientific meetings in order to enable continuous discussion on several important topics. One of the topics is 'Recent Advances in Diagnosis and Treatment for Malignancies of the Gastrointestinal Tract'. Recent advances in diagnostic imaging have made it possible to detect early cancers in the gastrointestinal tract, which could be cured by less invasive treatment. On the other hand, owing to development of novel antitumor agents, prognosis of patients with advanced or metastatic cancers is getting better. In this review the contents of the core symposia are briefly summarized.

\section{Progress in Chemotherapy and Targeted Therapy for Gastrointestinal Malignancies (Core Symposium 1 at the 6 th Annual Meeting of JGA)}

A recent trend in Japan for resectable stage II/III esophageal squamous cell cancer is neoadjuvant chemotherapy followed by surgery. Watanabe et al. [1] from

\section{KARGER}

Fax +41613061234 E-Mail karger@karger.ch www.karger.com
Hideo Baba, MD, PhD, FACS

Department of Gastroenterological Surgery

Graduate School of Medical Sciences, Kumamoto University

1-1-1 Honjo, Kumamoto 860-8556 (Japan)

Tel. +81 96373 5212, E-Mail hdobaba@ kumamoto-u.ac.jp 
Kumamoto University treated node-positive esophageal cancer with two courses of modified DCF (docetaxel, cisplatin and 5-fluorouracil) regimen as neoadjuvant or induction chemotherapy, and demonstrated an excellent treatment effect and sufficient downstaging. Induction chemotherapy is defined as the use of drug therapy as the initial treatment for patients presenting with advanced cancer that cannot be treated by other means. In order to improve the survival of esophageal cancer, establishment of multimodal treatment strategy, especially of neoadjuvant treatment, is essential.

Two important topics on chemotherapy for gastric cancer were adjuvant chemotherapy for resectable cancers and a new regimen for peritoneal dissemination. Emi et al. from Kyushu University demonstrated the strategy of clinical trials on the adjuvant and neoadjuvant chemotherapy, using S-1 plus docetaxel. They suggested that the poor compliance due to postoperative morbidity or disorders might be a major problem in the adjuvant chemotherapy. An efficacy of neoadjuvant chemotherapy for advanced cancer will be clarified in the near future. Ishigami et al. from Tokyo University reported a phase II trial of oral S-1 plus intraperitoneal paclitaxel for peritoneal metastases. They treated 18 patients and the response rate was $56 \%$ (95\% CI 32-79). Sixteen gastrectomies, including 13 curative resections, were carried out. Large-scale trials on such an effective regimen are expected to clarify the efficacy.

Recent advances in chemotherapy for colorectal cancer have improved the survival of unresectable or metastatic cases. As the response to chemotherapy varies among individuals, response predictors would be helpful to decide on a personalized treatment strategy. Ishizuka et al. from Dokkyo University investigated the possibility of Glasgow prognostic score in predicting response to FOLFILI or FOLFOX4, and demonstrated that the Glasgow prognostic score was a significant prognostic factor by multivariate analysis. Furukawa et al. from KKR Sapporo Medical Center demonstrated that skin toxicity was a predictive marker for response to cetuximab in Japanese patients as well as in Western countries.

Targeted therapy has become one of the key modalities for colorectal cancer. EGFR-KRAS signaling is especially a major target. Sugimoto et al. from Osaka Medical Center for Cancer and Cardiovascular Diseases investigated the efficacy of cetuximab in Japanese patients with K-ras wild-type advanced or metastatic colorectal cancer, and revealed that the efficacy of cetuximab in Japanese patients was comparable to those reported in the Western countries. Kimura et al. from Tokushima University re- ported the significance of a high sensitivity analysis of KRAS and BRAF in predicting the response to cetuximab. The two-step PCR-RFLP method increased the detection rate of both KRAS and BRAF mutations, and thus may help in selecting patients who benefit from cetuximab.

Rapid progress has also been observed in the strategy for colorectal liver metastasis. Sato et al. from Kitazato University demonstrated the improved survival after the introduction of FOLFOX. They demonstrated that neoadjuvant chemotherapy using recent new drug regimens may improve the survival of colorectal liver metastasis, although a further large-scale analysis is needed.

\section{Recent Advances in Diagnosis and Treatment for Esophageal Cancer - From Early to Advanced Cancers (Core Symposium 1 at the 7th Annual Meeting of JGA)}

Esophagectomy is a surgery involving a lot of surgical stress. Therefore, a demand for non-surgical management of superficial esophageal cancer is increasing. Yamada et al. from Osaka University demonstrated results of their basic strategy; endoscopic mucosal resection (EMR)/endoscopic submucosal dissection (ESD) for mucosal cancer (T1a) and chemoradiotherapy (CRT) for submucosal cancer (T1b). They revealed that survival after EMR/ESD for Tla cancer was excellent and prognosis after CRT for T1b was comparable to esophagectomy. Iizuka et al. from Toranomon Hospital demonstrated their own results of ESD for superficial esophageal cancer invading into the muscularis mucosa without nodal involvement. They decided the necessity of additional treatment strategy based on the pathologic results of ESD. As ESD followed by surgery or CRT can be performed safely and long-term survival of these patients was favorable, their strategy seemed to be feasible. Yamaguchi et al. from Nagasaki University presented the efficacy of ESD and photodynamic therapy. They demonstrated that a prophylactic oral steroid significantly reduced the stenosis after ESD for lesions more than 3/4 circumferences. They also demonstrated photodynamic therapy as a useful alternative for local recurrence after CRT.

Yamamoto et al. from Osaka Medical Center for Cancer and Cardiovascular Diseases retrospectively analyzed prognoses of stage I disease treated either by surgery or CRT, and demonstrated that the long-term survival of patients treated by CRT was comparable with those treated by surgery. They concluded that CRT can be an alternative for esophagectomy in stage I esophageal cancer. 
Less invasive surgery is another approach to reduce the surgical risk. Matsumoto et al. from Kawasaki Medical School demonstrated results of transhiatal esophagectomy for superficial esophageal cancer or advanced lower esophageal cancer. They concluded that the less invasive surgery can be indicated for selected patients.

Prediction of therapeutic response is a matter of the greatest importance. Watanabe et al. from Kumamoto University reported the correlation between microRNA expression in the pretreatment biopsy samples and response to induction DCF. Akutsu et al. from Chiba University demonstrated that high COX2 expression in the pretreatment biopsy samples can be a biomarker for resistance to CRT. Minashi et al. from National Cancer Center East Hospital demonstrated an efficacy of gene profiling in predicting prognosis of patients with stage II/ III esophageal cancer treated by CRT. These efforts might enable tailor-made treatment for esophageal cancer in the near future.

Alteration of chemotherapeutic regimen is another approach to improve the response to CRT. Kimura et al. from Tokushima University reported the results of CRT with a novel regimen consisted of irradiation combined with 5-FU/nedaplatin and indicated that this regimen may improve the survival. Large-scale trials are needed to establish the more efficient drug combination.

Salvage esophagectomy is an effective treatment for patients with remnant or recurrent diseases after definitive chemoradiotherapy, although high morbidity and mortality has been reported. Takemura et al. from Hyogo College of Medicine demonstrated their own experiences on salvage esophagectomy. They could prevent hospital mortality by limiting the lymph node dissection. They also revealed that the long-term survival depended on the stage before CRT.

\section{Discussion}

The contents of these two core symposia illustrated two directions in the management of gastrointestinal malignancies, one of which is a less invasive treatment for early diseases and another is a multimodal treatment strategy for advanced or metastatic diseases. Although there were few debates on the advances in diagnosis of gastrointestinal tumors in these symposia, an accurate diagnosis is essential for making appropriate treatment strategy. Progress in the diagnosis, such as recent endoscopic technology for accurate diagnosis of early cancers and usefulness of positron emission tomography for ad- vanced cancers, will be discussed in the future symposium.

ESD has become one of the major treatment modalities for early gastrointestinal cancers [2]. Efforts to extend the indication are in progress and will be good news for patients. CRT is not only a less invasive treatment but also contributes to organ preservation. However, the outcome of patients refractory to CRT is pessimistic. Therefore, predictive parameters for the effect of CRT should be established, while safety of salvage treatment should be assured. As a less invasive treatment, significance of minimally invasive surgery, including laparoscopic or thoracoscopic surgery, should be clarified in a future symposium.

In Japan the surgeon's efforts have been focused on complete removal of regional lymph nodes. D2 dissection for gastric cancer has been proven to have survival benefit in a long-term follow-up of a Dutch trial [3]. Similarly, the number of dissected lymph nodes has been reported to correlate with the prognosis of colon cancer [4]. These findings indicate that lymph node dissection contributes to prolonged survival. On the other hand, several randomized control trials which compared prognosis of patients between standard and extended lymphadenectomy failed to demonstrate the survival benefit $[5$, 6], suggesting that the effect of lymph node dissection is limited. Adjuvant chemotherapy might be promising to overcome the limitation.

The effect of adjuvant chemotherapy has already been proven in gastric [7], colon [8] and pancreatic cancers [9]. In esophageal cancer the survival benefit of neoadjuvant chemotherapy compared to adjuvant chemotherapy has been proven in Japanese patients [10]. Benefits of neoadjuvant chemotherapy compared to adjuvant chemotherapy include good compliance for the treatment and increased curability in surgery. However, there is a risk to disease progression in cases refractory to chemotherapy. Therefore, biomarkers to predict response to chemotherapy are needed to decide on an appropriate treatment strategy for each patient.

\section{Conclusions}

Less invasiveness and multidisciplinary approach are the two major key words for the management of gastrointestinal malignancies. Efforts in translational research as well as in clinical studies will deliver less invasive and more effective treatment for gastrointestinal cancers. 


\section{Acknowledgement}

We express our sincere appreciation to all authors who contributed to the core symposia.

\section{Disclosure Statement}

The authors declare that no financial conflict or conflict of interest exists in relation to the content of the article.

\section{References}

1 Watanabe M, Nagai Y, Kinoshita K, Saito S, Kurashige J, Karashima R, Hirashima K, Sato N, Imamura Y, Hiyoshi Y, Baba Y, Iwagami S, Miyamoto Y, Iwatsuki M, Hayashi $\mathrm{N}$, Baba H: Induction chemotherapy with docetaxel/cisplatin/5-fluorouracil for patients with node-positive esophageal cancer. Digestion 2011;83:146-152.

- 2 Oda I, Gotoda T, Hamanaka H, Eguchi T, Saito Y, Matsuda T, Bhandari P, Emura F, Saito D, Ono H: Endoscopic submucosal dissection for early gastric cancer: technical feasibility, operation time and complications from a large consecutive series. Dig Endosc 2005; 17:54-58.

- 3 Songun I, Putter H, Kranenbarg EMK, Sasako M, van Velde CJH: Surgical treatment of gastric cancer: 15 -year follow-up results of the randomized nationwide Dutch D1D2 trial. Lancet Oncol 2010;11:439-449.

$\checkmark 4$ Le Voyer TE, Sigurdson ER, Hanlon AL, Mayer RJ, Macdonald JS, Catalano PJ, Haller DG: Colon cancer survival is associated with increasing number of lymph nodes analyzed: a secondary survey of intergroup trial INT0089. J Clin Oncol 2003;21:2912-2919.
5 Sasako M, Sano T, Yamamoto S, Kurokawa Y, Nashimoto A, Kurita A, Hiratsuka M, Tsujinaka T, Kinoshita T, Arai K, Yamamura Y, Okajima K, for the Japan Clinical Oncology Group: D2 lymphadenectomy alone or with para-aortic nodal dissection for gastric cancer. N Engl J Med 2008;359:453-462.

6 Pedrazzoli S, DiCarlo V, Dionigi R, Mosca F, Pederzoli P, Pasquali C, Kloppel G, Dhaene $\mathrm{K}$, Michelassi F, and the Lymphadenectomy Study Group: Standard versus extended lymphadenectomy associated with pancreatoduodenectomy in the surgical treatment of adenocarcinoma of the head of the pancreas. A multicenter, prospective, randomized study. Ann Oncol 1998;228:508-517.

-7 Sakuramoto S, Sasako M, Yamaguchi T, Kinoshita T, Fujii M, Nashimoto A, Furukawa H, Nakajima T, Ohashi Y, Imamura H, Higashino M, Yamamura Y, Kurita A, Arai $\mathrm{K}$, for the ACTS-GC Group: Adjuvant chemotherapy for gastric cancer with S-1, an oral fluoropyrimidine. N Engl J Med 2007; 357:1810-1820.
8 Andre T, Boni C, Navarro M, Tabernero J, Hickish T, Topham C, Bonetti A, Clingan P, Bridgewater J, Rivera F, de Gramont A. Improved overall survival with oxaliplatin, fluorouracil and leucovorin as adjuvant treatment in stage II or III colon cancer in the MOSAIC trial. J Clin Oncol 2009;27:31093116.

-9 Ueno H, Kosuge T, Matsuyama Y, Yamamoto J, Nakao A, Egawa S, Doi R, Monden M, Hatori T, Tanaka M, Shimada M, Kanemitsu K. A randomized phase III trial comparing gemcitabine with surgery-only in patients with resected pancreatic cancer: Japanese Study Group of Adjuvant Therapy for Pancreatic Cancer. Br J Cancer 2009;101:908915.

10 Igaki H, Kato H, Ando N, Shimada M, Shimizu H Nakamura T, Ozawa S, Yabusaki H, Aoyama N, Kurita A, Fukuda H: A randomized trial of postoperative chemotherapy with cisplatin and 5-fluorouracil versus neoadjuvant chemotherapy for clinical stage II/ III squamous cell carcinoma of the thoracic esophagus (abstract). ASCO Annual Meeting, 2008 . 